\title{
Biography—David Menter
}

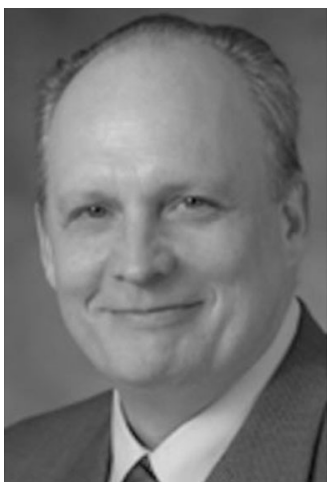

David Menter, Ph.D., is an assistant professor of Gastrointestinal Medical Oncology at the MD Anderson Cancer Center. He trained with Kenneth Honn, Ph.D., and Lawrence Marnett, Ph.D., in the study of prostaglandins in the molecular and cellular biology of cancer. He published many of the early seminal papers on the role of platelets in cancer metastasis. His experience with prostaglandins and other bioactive lipids extends to a chapter in Prostaglandins and Cancer: The First International Conference, published in 1981. Drs. Honn and Menter recently contributed to the second edition of Platelets in Thrombotic and Non-Thrombotic Disorders, edited by Paolo Gresele, Neal Kleiman, José Lopez, and Clive Page. Dr. Menter is an expert in the study of prostaglandins; cyclooxygenase-1 and -2; prostaglandin $\mathrm{E}_{2}$-mediated and CSN6 signal transduction; and inflammation and carcinogenesis.

Dr. Menter was awarded a National Institutes of Health postdoctoral fellowship in Human Genetics. He was then promoted to assistant professor and worked with Scott Lippman, M.D., to establish the Department of Clinical Cancer Prevention in the Division of Cancer Prevention, and then with Dr. Lippman and
Waun Ki Hong, M.D., to develop the Lung Cancer Biomarkers Core Program in the Department of Thoracic/Head and Neck Medical Oncology. Several years later, the trio would work together once more to provide the infrastructure for the Biomarker-integrated Approaches of Targeted Therapy for Lung Cancer Elimination (BATTLE) trials. He was first to study the role of neurotrophins in brain cancer metastasis and to implement the use of CreERT conditionally targeted mice to examine the role of selenium and selenoproteins in prostate cancer. More recently, Dr. Menter worked with Drs. Raymond DuBois, M.D., Ph.D., and Ernest Hawk, M.D., M.P.H, where he gained extensive experience with NSAIDs COXIBs and aspirin and colorectal cancer biology. He also developed experience with development of nanoparticle delivery vehicles for the treatment of GI cancers and the development of next-generation animal models of GI cancers. He helped found ACF-Pharmaceuticals LLP and serves on its executive board as a member and as chief scientific officer. He is involved in establishing the Center for Professional Development and Entrepreneurship as part of an MD Anderson program to support the innovation and commercialization of new technology by helping provide curricular offerings, collaborative networks, and professional guidance.

Dr. Menter serves as scientific director of the Colorectal Cancer-Moon Shot. He and Scott Kopetz, M.D., Ph.D., are investigating the importance of colorectal cancer consensus molecular subtypes (CMS) as part of their Integromics program, which utilizes and integrates next-generation sequencing and other molecular data to understand the four different CMS subtypes of colorectal cancer. He also is developing novel mouse models to better understand the role of platelets in cancer and metastasis in collaboration with Anil Sood, M.D., who discovered the systemic tumor-IL6: liverthrombopoeitin: bone marrow platelet overproduction loop that drives Trousseau's syndrome in cancer. 